\title{
Impact of focal cortical dysplasia Type IIIa on seizure outcome following anterior mesial temporal lobe resection for the treatment of epilepsy
}

\author{
Lasse Dührsen, MD, ${ }^{1}$ Thomas Sauvigny, MD, ${ }^{1}$ Patrick M. House, MD, ${ }^{2}$ Stefan Stodieck, MD, ${ }^{2}$ \\ Brigitte Holst, MD, ${ }^{3}$ Jakob Matschke, MD, ${ }^{4}$ Gerhard Schön, MSc, ${ }^{5}$ Manfred Westphal, MD, ${ }^{1}$ and \\ Tobias Martens, MD'
}

Departments of ${ }^{1}$ Neurosurgery, ${ }^{3}$ Neuroradiology, ${ }^{4}$ Neuropathology, and ${ }^{5}$ Medical Biometry and Epidemiology, University Medical Center Hamburg-Eppendorf; and 2Department of Neurology and Epileptology, Hamburg Epilepsy Center, Hamburg, Germany

\begin{abstract}
OBJECTIVE Temporal lobe epilepsy (TLE) is the most common type of pharmacoresistant focal epilepsy, for which anterior mesial temporal lobe resection (AMTLR) is a treatment option. Focal cortical dysplasia Type Illa (FCD IIla), a developmental lesion resulting from defects in neuronal formation and migration into the temporal pole (FCD I) combined with hippocampal sclerosis (HS), can be a neuropathological finding. In this study, the authors investigate the impact of FCD Illa on seizure outcome in patients with TLE who underwent AMTLR.

METHODS The authors performed a retrospective analysis of all patients with TLE who underwent AMTLR at their institution between June 2011 and April 2014. Histopathological analysis was used to determine whether patients had HS together with FCD I (FCD IIla) or HS alone. The groups were compared with regard to age, sex, years of epilepsy, and seizure outcome using the Engel classification.
\end{abstract}

RESULTS A total of 51 patients with TLE underwent AMTLR at the authors' institution. FCD Illa was diagnosed in 13 cases. The patients experienced seizures for a mean duration of 31.1 years. The mean length of follow-up after the procedure was 18 months. All patients with FCD Illa had a favorable seizure outcome (Engel Class I or II) compared with $71 \%$ of the patients with no pathological findings in the temporal pole $(p<0.01)$.

CONCLUSIONS Patients with histopathologically proven FCD Illa had a significantly better seizure outcome after AMTLR than patients with HS alone. Further effort should be made during presurgical evaluation to detect FCD Illa so that the most suitable resection technique can be chosen and postoperative seizure outcome can be predicted for patient counseling.

https://thejns.org/doi/abs/10.3171/2017.2.JNS161295

KEY WORDS epilepsy surgery; FCD IIla; anterior mesial temporal lobe resection; focal cortical dysplasia

$\mathrm{T}$ emporal lobe epilepsy (TLE) is the most common cause of pharmacoresistant focal epilepsy, for which surgery is the treatment of choice. ${ }^{6}$ Whether selective amygdalohippocampectomy (selAH) or anterior mesial temporal lobe resection (AMTLR) is the preferred procedure is an ongoing and controversial debate, especially with regard to the respective efficacy in seizure control. ${ }^{13,16,21}$ A general decline in verbal memory function after temporal lobe surgery in the dominant hemisphere often occurs, irrespective of the technique employed..$^{13,18}$ Yet the criteria for decision making regarding the choice of selAH or AMTLR are not clear. However, even when the same operative technique is used, seizure outcome differs for various reasons, ${ }^{26}$ including underlying subtle pathologies like focal cortical dysplasias (FCDs) or adjacent epileptogenic zones. ${ }^{1}$

In 2011, Blümcke et al. introduced a new classification system for FCDs. ${ }^{3}$ Here, in particular, FCD Type IIIa

ABBREVIATIONS AMTLR = anterior mesial temporal lobe resection; EEG = electroencephalography; FCD IIla = focal cortical dysplasia Type IIla; HS = hippocampal sclerosis; ILAE = International League Against Epilepsy; selAH = selective amygdalohippocampectomy; TLE = temporal lobe epilepsy.

SUBMITTED May 20, 2016. ACCEPTED February 9, 2017.

INCLUDE WHEN CITING Published online July 28, 2017; DOI: 10.3171/2017.2.JNS161295. 
(FCD IIIa) - the combination of hippocampal sclerosis (HS) and FCD Type I in the adjacent temporal pole-is of particular interest. However, it is imperative that FCD IIIa is not considered as a dual pathology. It is currently unclear if FCD IIIa is associated with a distinct pathological entity. This is suggested by the hypothesis that HS in FCD IIIa is a product of ongoing seizures triggered by the FCD, thereby forming an epileptic unit. ${ }^{3}$ If this is not the case, FCD and HS should be considered as 2 epileptic foci that are independent of each other.

There is indication that reelin, a glycoprotein responsible for neuronal migration and architecture in the hippocampus and cortical layers, plays an important role in a subset of FCD IIIa. ${ }^{19}$ There are also reports of familial TLE with FCD IIIa that indicate a genetic cause. ${ }^{8}$ These findings would support the hypothesis that FCD IIIa is a single epileptic unit and therefore an individual disease pattern. However, there is no clear clinical picture that would render the diagnosis possible. In particular, semiology and electrophysiological patterns show no substantial differences between HS alone and HS together with FCD IIIa. ${ }^{15,22}$ Furthermore, FCD IIIa is often too subtle to be observed on MRI. Morphometric analysis based on T1and T2-weighted MRI volume data sets has proved to be a helpful diagnostic tool for the detection of FCDs. ${ }^{12}$

The underlying pathologies, such as FCD IIIa, in the temporal lobe often escape diagnostic detection due to a lack of sufficient diagnostic tools. Hence, they are frequently diagnosed histopathologically. ${ }^{17}$ If pathologies like FCD IIIa represent a single epileptic unit and not 2 individual foci, it is crucial to determine which surgical approach should be used. To investigate the impact of FCD IIIa on seizure outcome, we analyzed patients who underwent AMTLR.

\section{Methods}

\section{Patient Characteristics and Study Design}

All patients with pharmacoresistant TLE who underwent AMTLR between June 2011 and April 2014 and had histologically proven HS or HS together with FCD I (FCD IIIa) were included for evaluation. Patients with evidence of tumor, vascular malformation, and/or extratemporal seizure foci were excluded. Presurgical evaluation, performed at the Hamburg Epilepsy Center, comprised anamnesis of seizure semiology, video electroencephalography (EEG) monitoring, 3-T MRI, neuropsychological testing, and SPECT and/or PET; language lateralization was determined by functional transcranial Doppler ultrasonography results. ${ }^{10}$ All patients underwent surgery at the section for epilepsy surgery at the Department of Neurosurgery, University Medical Center Hamburg-Eppendorf. Postoperative MRI was performed during their hospital stay. The patients underwent follow-up through clinical investigations and EEG at the Hamburg Epilepsy Center and in the outpatient clinic at the Department of Neurosurgery, University Medical Center Hamburg-Eppendorf. Seizure outcome was evaluated according to the Engel classification system. ${ }^{7}$ Analysis of correlative clinical data was performed retrospectively by chart review. Informed consent for clinical data evaluation was provid- ed by all patients. The decision for AMTLR was made by epileptologists and neurosurgeons together. Our epilepsy data register is approved by the local ethics committee at the medical council of the state of Hamburg (Ethik-Kommission der Ärztekammer Hamburg).

\section{AMTLR Technique}

A temporal-accentuated pterional approach was used. After identifying and opening the sylvian fissure, the temporal pole was removed en bloc and sent for histological evaluation. Subsequently, the temporal horn of the lateral ventricle was opened, and the anterior part of the hippocampus was exposed. The temporomesial structures including the hippocampus, gyrus parahippocampalis, and amygdala were then removed via subpial resection. Then, the oculomotor nerve, choroid plexus, and posterior cerebral artery in front of the brainstem were exposed, serving as landmarks. An example of the performed resections is shown in Fig 1.

\section{Histopathological Diagnosis}

Tissue was fixed in $10 \%$ formalin for at least 6 hours; to obtain maximal penetration of the fixative, larger samples were sliced into approximately 5-mm-thick sections immediately after the specimens arrived in the neuropathology laboratory. Before being embedded in paraffin, the specimens were carefully oriented in order to obtain microscopic sections perpendicular to the pial surface. Each surgical specimen was completely embedded. Paraffin sections of $4 \mu \mathrm{m}$ thickness from each block underwent immunohistochemical staining as suggested by Blümcke et al. ${ }^{3}$ The stains included H \& E, van Gieson's stain, Turnbull's stain, Luxol-PAS, Masson's trichrome, and Bodian's or Bielschowsky's stain following standard laboratory pro-
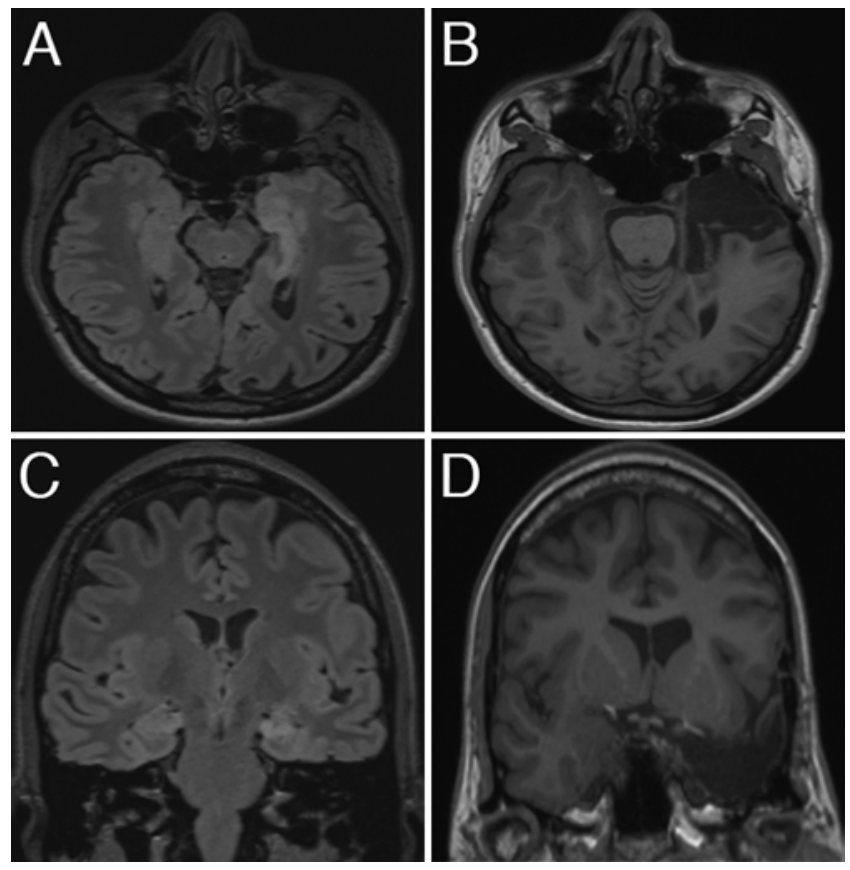

FIG. 1. Example of pre- ( $A$ and $C$ ) and postoperative ( $B$ and $D$ ) axial and coronal MR images after AMTLR on the left side. 


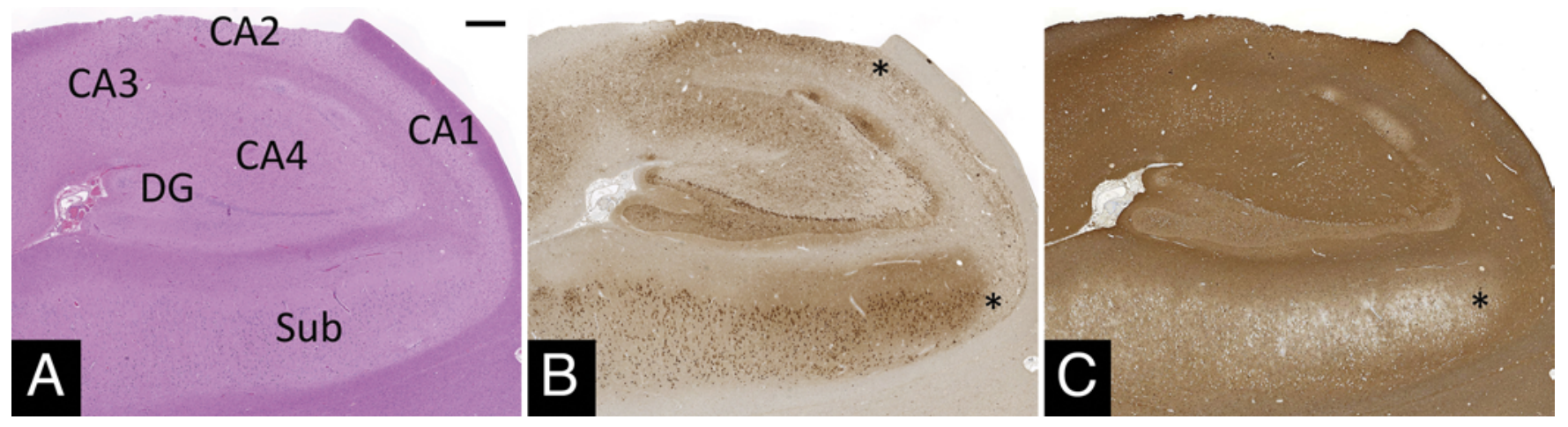

FIG. 2. Histological analysis of HS according to ILAE Type 1 showing severe pyramidal cell loss predominantly in sectors CA1 and CA4 (A; and between the asterisks, B) accompanied by dense fibrillary gliosis with sharp cutoff within the subiculum (asterisk, C). $H \& E(A)$, and Neu-N (B) and GFAP (C) immunohistochemistry. Bar =500 $\mu \mathrm{m}$. DG = dentate gyrus; Sub = subiculum.

cedures. ${ }^{3}$ Furthermore, sections from each paraffin block were stained using an automated Ventana HX IHC system (Ventana-Roche Medical systems) according to the manufacturer's instructions. In each case, immunohistochemistry included NeuN (Chemicon), MAP2c (Sigma), GFAP (DAKO), and neurofilament (DAKO). In selected cases, further staining for CD34 (DAKO), vimentin (DAKO), or Ki-67 (NeoMarkers) was done. The pathomorphological diagnosis of HS, temporal lobe sclerosis, and FCD IIIa was made according to the most recent international con- sensus classification of the International League Against Epilepsy (ILAE) 2,3,24 (Figs. 2 and 3).

\section{Imaging}

MR images were acquired using a 3.0-T MR scanner (Skyra, Siemens). The MRI protocol for preoperative imaging involved T2-weighted space dark fluid, T1-weighted MP-RAGE (magnetization-prepared rapid gradient-echo), and FLAIR volume data sets. Likewise, coronal T2-weight-
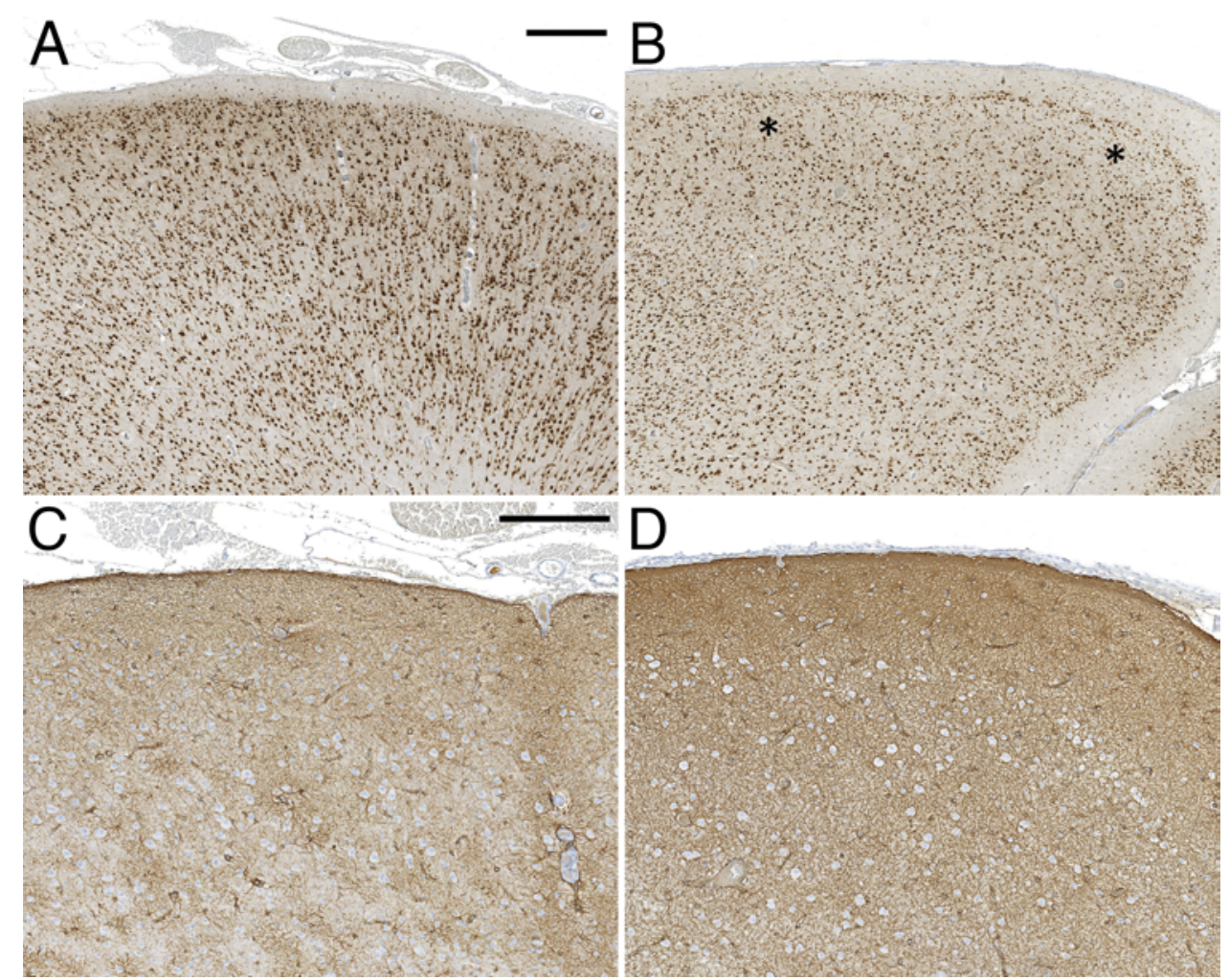

FIG. 3. Histological analysis of temporal lobe sclerosis with mild neuronal loss from layers II and III with cellular crowding of outer parts of layer II (asterisks in B) accompanied by diffuse laminar gliosis (D). Adjacent normal hexalaminar isocortex from the same specimen (A) without relevant gliosis (C). Immunohistochemistry for NeuN (A and B) and for GFAP (C and D). Bar = 500 $\mu \mathrm{m}(\mathrm{A}$, also applies to B); bar $=250 \mu \mathrm{m}(C$, also applies to $D)$. 
ed turbo spin echo, coronal T1-weighted turbo inversion recovery, and axial proton density/T2-weighted data sets were acquired. For enhanced FCD detection, the patients' MR images were additionally postprocessed at the Hamburg Epilepsy Center by means of morphometric MRI analysis using a fully automated script (MATLAB) commonly used for FCD detection, as described elsewhere. ${ }^{13}$ The required $\mathrm{T} 1$ and $\mathrm{T} 2$ normal databases are scanner- and sequence-specific and in everyday clinical use for morphometric MRI analyses at the Hamburg Epilepsy Center. ${ }^{11,12,14}$

\section{Statistical Analysis}

The correlation of histopathological diagnosis, seizure outcome, duration of epilepsy, and bilateral convulsive seizures was assessed using bivariate and multivariate analyses. Further statistical analyses were performed using Fisher's exact test and t-test. A 5\% significance level was accepted for hypothesis testing. All analyses were carried out using the $\mathrm{R}$ project for statistical computing software, version 3.2.2.

\section{Results}

A total of 51 patients with pharmacoresistant TLE underwent AMTLR. The sex distribution was nearly equal (24 males and 27 females), and patients ranged in age from 20 to 69 years. The onset of epilepsy occurred in patients between the ages of 0 and 48 years. The patients experienced seizures on average for 31.9 years (range 5-66 years). Ictal onset was identified on the left side in 24 patients $(47.1 \%)$ and on the right side in 27 patients $(52.9 \%)$. Thirty-seven patients $(72.5 \%)$ experienced secondary generalization of their seizures. Thirty-four patients $(66.7 \%)$ were undergoing their first surgical intervention for epilepsy. The remaining 17 patients $(33.3 \%)$ were undergoing their second procedure because of persistent seizures; 13 of these patients had been treated initially with selAH and 4 with AMTLR. Speech lateralization was detected in the right hemisphere in 5 patients $(9.8 \%)$ and in the left side in 46 patients $(90.2 \%)$. The MRI scans revealed morphological characteristics for HS in all cases. Based on MRI findings, there was no suspicion of FCD-related changes in the temporal pole. Postprocessing of the preoperative MR images revealed FCD IIIa in 3 cases. However, findings were positive for FCD IIIa on neuropathological examination in 13 patients $(25.5 \%)$, which was found in the temporal pole in each case. The remaining 38 patients (74.5\%) had HS alone.

Outcome according to the Engel classification system was analyzed 12 months postoperatively $(n=45 ; 6$ patients were lost to follow-up). Thirty-two patients (71.1\%) were free of disabling seizures, of whom 30 were in Engel Class I and 2 were in Engel Class II, thus having a favorable outcome. Thirteen patients $(28.9 \%)$ continued to suffer from seizures and were categorized as having a poor outcome (Engel Classes III and IV). A favorable seizure outcome was significantly affected by the finding of FCD IIIa ( $\mathrm{n}=$ 13, 18.3\%; Fisher's exact test, $p=0.0087$; Fig. 4).

Furthermore, we examined whether the duration of epilepsy or the presence of bilateral convulsive seizures might predict seizure outcome after 12 months. A multi-

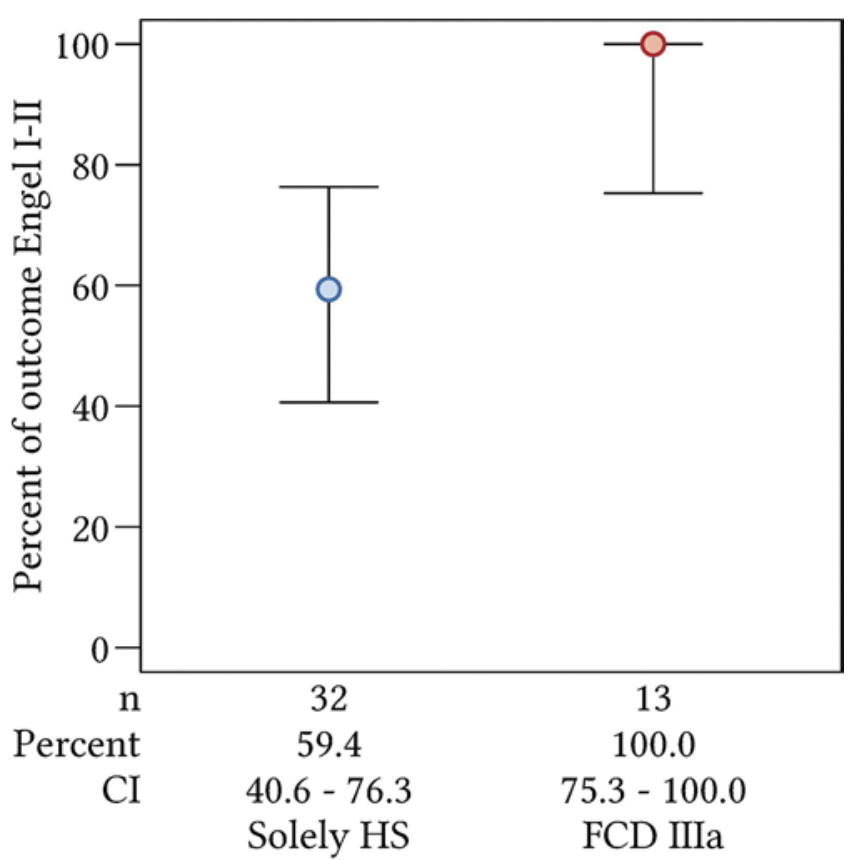

FIG. 4. Patients with FCD IIla and HS had a significantly better seizure outcome than those with solely hippocampal sclerosis. The circles indicate the means, and the error bars represent the SD. Figure is available in color online only.

variate analysis showed that there was no significant effect on seizure outcome in our series (Fig. 5).

\section{Discussion}

In this study, we showed that patients with pharmaco-

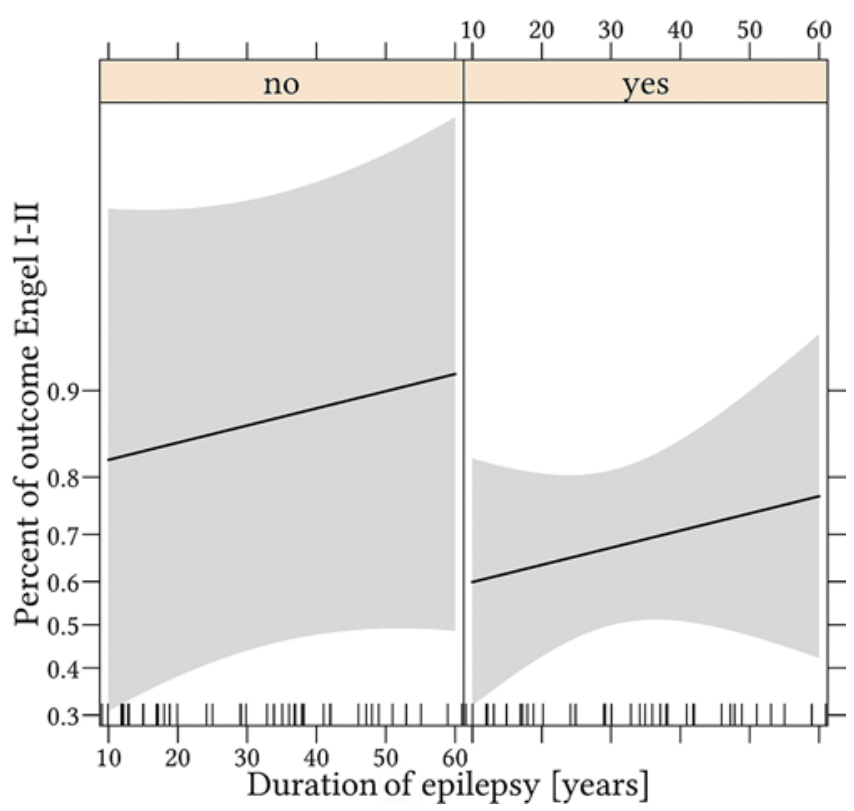

FIG. 5. Effect plot. Seizure outcome was not influenced by bilateral convulsive seizures or by years of epilepsy. "No" and "yes" indicate the absence and presence of bilateral convulsive seizures, respectively. The lines represent the probability, and the gray areas indicate the $95 \% \mathrm{Cl}$. Figure is available in color online only. 
resistant TLE are more likely to become seizure free after AMTLR if FCD I together with HS (FCD IIIa) rather than HS alone is found histopathologically. Multivariate analysis revealed that the duration of epilepsy and the presence of bilateral convulsive seizures did not influence seizure outcome. Conventional review of preoperative MRI scans did not reveal any FCD IIIa; however, postprocessing of these MR images detected that 3 patients had FCD IIIa. Two patients who had previously undergone selAH underwent AMTRL because of persistent seizures, and results of histopathological analysis were positive for FCD IIIa.

Because of a lack of reliable and sufficient diagnostic tools, FCD III is rarely diagnosed preoperatively and is diagnosed based on histopathological findings. Temporal pole abnormalities could be identified before surgery in some cases only when MRI data were postprocessed by morphometric analysis. However, the relevance of FCD IIIa for seizure outcome suggests improving the sensitivity and specificity of this technique to achieve better patient counseling in terms of efficacy of the procedure. The lack of reliable preoperative diagnostic tools makes it difficult to decide which resection technique should be used when it comes to surgery.

Seizure duration and the presence of bilateral convulsive seizures did not influence seizure outcome after AMTLR in our series. This precludes an effect on the seizure outcome in our study group.

Recent neuropathological data support that FCD IIIa might be an epileptic unit unto itself. ${ }^{9}$ Before the latest FCD classification was introduced, very good seizure outcomes were seen in patients with both HS and ipsilateral temporal lobe sclerosis when both pathologies were removed. ${ }^{23}$ Presurgical evaluation with MRI proved unsuccessful in the detection of FCD IIIa, which is in line with reports from other authors. ${ }^{25}$ Therefore, finding and establishing reproducible diagnostic tools for detecting FCD IIIa is of great importance. The use of morphometric MRI analysis seems to be a promising and helpful tool for presurgical evaluation, even though in our study only 3 of 13 histopathologically proven cases of FCD IIIa were identified preoperatively on MRI postprocessing. ${ }^{25}$ As recently reported, a lack of magnetic field strength with standard MRI might result in negative findings for FCD IIIa. ${ }^{20}$ Likewise, the preoperative findings of undefined temporal abnormalities on standard MRI seem to have no influence on seizure outcome. ${ }^{4}$ Another aspect that is still underdeveloped is the need to look for specific clinical patterns in seizures and on EEG to differentiate the underlying causes, even though neither we nor others have been able to find significant differences in electroclinical features. ${ }^{15}$

Finally, a question that remains is whether FCD (Type I) in the temporal pole is responsible for the development of ipsilateral HS in the context of FCD IIIa. There are hints of an underlying genetic cause of temporal FCD IIIa as well as for the existence of regulatory proteins affecting the pathogenesis of FCD IIIa. ${ }^{5,19}$ HS might develop as a consequence of early seizure onset in life due to undiscovered FCD IIIa. Even though the 2 groups did not differ significantly in terms of age at epilepsy onset, there seems to be a trend. Eight patients (61.5\%) harboring FCD
IIIa were between 9 and 14 years old at epilepsy onset, whereas in the HS group the age distribution was much wider (range 0-48 years). This can be interpreted as a common clinical feature of FCD IIIa and would underline the theory of a congenital disease. Taken together, these results suggest that the existence of FCD I in addition to HS (FCD IIIa) compared with HS alone is associated with a better seizure outcome after AMTLR.

\section{Conclusions}

This was a single-center retrospective study with a relatively short follow-up period. Nevertheless, we were able to show that poor seizure outcomes after selAH could be due to undetected FCD IIIa in the temporal pole, a condition that might occur more often than expected. Further effort should be made during presurgical evaluation to detect FCD IIIa in order to facilitate both the choice of the suitable resection technique and the prediction of postoperative seizure outcome for patient counseling.

\section{References}

1. Barba C, Rheims S, Minotti L, Guénot M, Hoffmann D, Chabardès $\mathrm{S}$, et al: Temporal plus epilepsy is a major determinant of temporal lobe surgery failures. Brain 139:444451,2016

2. Blümcke I, Thom M, Aronica E, Armstrong DD, Bartolomei F, Bernasconi A, et al: International consensus classification of hippocampal sclerosis in temporal lobe epilepsy: a Task Force report from the ILAE Commission on Diagnostic Methods. Epilepsia 54:1315-1329, 2013

3. Blümcke I, Thom M, Aronica E, Armstrong DD, Vinters HV, Palmini A, et al: The clinicopathologic spectrum of focal cortical dysplasias: a consensus classification proposed by an ad hoc Task Force of the ILAE Diagnostic Methods Commission. Epilepsia 52:158-174, 2011

4. Di Gennaro G, D’Aniello A, De Risi M, Grillea G, Quarato PP, Mascia A, et al: Temporal pole abnormalities in temporal lobe epilepsy with hippocampal sclerosis: Clinical significance and seizure outcome after surgery. Seizure 32:84-91, 2015

5. Donkels C, Pfeifer D, Janz P, Huber S, Nakagawa J, Prinz $\mathrm{M}$, et al: Whole transcriptome screening reveals myelination deficits in dysplastic human temporal neocortex. Cereb Cortex 27:1558-1572, 2017

6. Engel J Jr, McDermott MP, Wiebe S, Langfitt JT, Stern JM, Dewar S, et al: Early surgical therapy for drug-resistant temporal lobe epilepsy: a randomized trial. JAMA 307:922930, 2012

7. Engel J Jr, Van Ness PC, Rasmussen TB, Ojemann LM: Outcome with respect to epileptic seizures, in Engel J Jr (ed): Surgical Treatment of the Epilepsies, ed 2. New York: Raven Press, 1993, pp 609-621

8. Fabera P, Krijtova H, Tomasek M, Krysl D, Zamecnik J, Mohapl M, et al: Familial temporal lobe epilepsy due to focal cortical dysplasia type IIIa. Seizure 31:120-123, 2015

9. Fauser S, Häussler U, Donkels C, Huber S, Nakagawa J, Prinz M, et al: Disorganization of neocortical lamination in focal cortical dysplasia is brain-region dependent: evidence from layer-specific marker expression. Acta Neuropathol Commun 1:47, 2013

10. House PM, Brückner KE, Lohmann HH: Presurgical functional transcranial Doppler sonography (fTCD) with intravenous echo enhancing agent SonoVue enables determination of language lateralization in epilepsy patients with poor temporal bone windows. Epilepsia 52:636-639, 2011 
11. House PM, Holst B, Lindenau M, Voges B, Kohl B, Martens $\mathrm{T}$, et al: Morphometric MRI analysis enhances visualization of cortical tubers in tuberous sclerosis. Epilepsy Res 117:2934, 2015

12. House PM, Lanz M, Holst B, Martens T, Stodieck S, Huppertz HJ: Comparison of morphometric analysis based on T1 and T2-weighted MRI data for visualization of focal cortical dysplasia. Epilepsy Res 106:403-409, 2013

13. Hu WH, Zhang C, Zhang K, Meng FG, Chen N, Zhang JG: Selective amygdalohippocampectomy versus anterior temporal lobectomy in the management of mesial temporal lobe epilepsy: a meta-analysis of comparative studies. J Neurosurg 119:1089-1097, 2013

14. Huppertz HJ, Grimm C, Fauser S, Kassubek J, Mader I, Hochmuth A, et al: Enhanced visualization of blurred graywhite matter junctions in focal cortical dysplasia by voxelbased 3D MRI analysis. Epilepsy Res 67:35-50, 2005

15. Johnson AM, Sugo E, Barreto D, Cunningham AM, Hiew CC, Lawson JA, et al: Clinicopathological associations in temporal lobe epilepsy patients utilising the current ILAE focal cortical dysplasia classification. Epilepsy Res 108:13451351,2014

16. Josephson CB, Dykeman J, Fiest KM, Liu X, Sadler RM, Jette N, et al: Systematic review and meta-analysis of standard vs selective temporal lobe epilepsy surgery. Neurology 80:1669-1676, 2013

17. Kim DW, Kim S, Park SH, Chung CK, Lee SK: Comparison of MRI features and surgical outcome among the subtypes of focal cortical dysplasia. Seizure 21:789-794, 2012

18. Lee TM, Yip JT, Jones-Gotman M: Memory deficits after resection from left or right anterior temporal lobe in humans: a meta-analytic review. Epilepsia 43:283-291, 2002

19. Marucci G, Farnedi A, Giulioni M: Reelin: a possible link between hippocampal sclerosis and cortical dyslamination in the setting of FCD type IIIa. Neurol Sci 33:1479-1481, 2012

20. Reeves C, Tachrount M, Thomas D, Michalak Z, Liu J, Ellis $\mathrm{M}$, et al: Combined ex vivo 9.4T MRI and quantitative histopathological study in normal and pathological neocortical resections in focal epilepsy. Brain Pathol 26:319-333, 2016

21. Schramm J: Temporal lobe epilepsy surgery and the quest for optimal extent of resection: a review. Epilepsia 49:12961307,2008

22. Spreafico R, Blümcke I: Focal cortical dysplasias: clinical implication of neuropathological classification systems. Acta Neuropathol 120:359-367, 2010

23. Tassi L, Garbelli R, Colombo N, Bramerio M, Lo Russo G,
Deleo F, et al: Type I focal cortical dysplasia: surgical outcome is related to histopathology. Epileptic Disord 12:181191,2010

24. Thom M, Eriksson S, Martinian L, Caboclo LO, McEvoy AW, Duncan JS, et al: Temporal lobe sclerosis associated with hippocampal sclerosis in temporal lobe epilepsy: neuropathological features. J Neuropathol Exp Neurol 68:928938, 2009

25. Wang ZI, Alexopoulos AV, Jones SE, Najm IM, Ristic A, Wong $\mathrm{C}$, et al: Linking MRI postprocessing with magnetic source imaging in MRI-negative epilepsy. Ann Neurol 75:759-770, 2014

26. West S, Nolan SJ, Cotton J, Gandhi S, Weston J, Sudan A, et al: Surgery for epilepsy. Cochrane Database Syst Rev (7):CD010541, 2015

\section{Disclosures}

The authors report no conflict of interest concerning the materials or methods used in this study or the findings specified in this paper.

\section{Author Contributions}

Conception and design: Dührsen, Martens. Acquisition of data: Dührsen, Sauvigny, House. Analysis and interpretation of data: Dührsen, Sauvigny, Holst, Matschke, Schön, Martens. Drafting the article: Dührsen, Matschke, Martens. Critically revising the article: Sauvigny, House, Westphal, Martens. Reviewed submitted version of manuscript: Dührsen, Stodieck, Holst, Westphal, Martens. Approved the final version of the manuscript on behalf of all authors: Dührsen. Statistical analysis: Dührsen, Schön. Study supervision: Martens.

\section{Supplemental Information}

\section{Previous Presentations}

Parts of this work were presented in a lecture at the 9th Annual Common Congress of the German, Austrian, and Swiss Leagues Against Epilepsy, Dresden, Germany, April 23, 2015.

\section{Correspondence}

Lasse Dührsen, Department of Neurosurgery, University Medical Center Hamburg-Eppendorf, Martinistraße 52, Hamburg 20246, Germany. email: 1.duehrsen@uke.de. 